\title{
Hidatidosis pulmonar. Presentación de un caso clínico
}

\author{
Alejandro Rogelio Canseco-Raymundo, $₫$ Fernando Flores-Trujillo, José de Jesús López-Luna, \\ Sergio Monraz-Pérez.
}

Instituto Nacional de Enfermedades Respiratorias Ismael Cosío Villegas, Ciudad de México.

Trabajo recibido: 06-X-2014; aceptado: 09-X-2014

\begin{abstract}
RESUMEN. La hidatidosis pulmonar es una parasitosis causada por formas larvarias de Echinococcus granulosus. En esta revisión se presenta el caso de un paciente femenino de 25 años de edad en convivencia directa con caninos, ovinos y aves silvestres, a quien le fue realizado exitosamente tratamiento médico y quirúrgico con resolución de complicaciones posoperatorias. La información en México sobre su prevalencia es limitada. Este reporte debe su importancia a la escasa frecuencia con que se ha documentado esta patología en el país.
\end{abstract}

Palabras clave: Echinococcus, hidatidosis pulmonar, quiste hidatídico.

ABSTRACT. Pulmonary hydatidosis is a parasitic disease caused by the larval stages of Echinococcus granulosus. We present a case of a 25 year old female patient, in direct contact with dogs, goats and poultry, in whom surgery and medical treatment, was successfully performed with resolution of postoperative complications. Information on its prevalence in Mexico is limited, so the importance of this report is due to the lack of information about this disease in our country.

Key words: Echinococcus, pulmonary hydatidosis, hydatid cist.

\section{INTRODUCCIÓN}

La hidatidosis o equinococosis es una parasitosis causada por formas larvarias del género Echinococcus: ${ }^{1-3}$ seis especies han sido reconocidas, pero sólo cuatro son de interés en salud pública: Echinococcus granulosus (E. granulosus), Echinococcus multilocularis, Echinococcus vageli y Echinococcus oligarthrus. ${ }^{4}$ Particularmente E. granulosus, como agente causal de la hidatidosis pulmonar en su forma quística, es un helminto que reside en el yeyuno de su huésped definitivo: perros y otros caninos, produciendo huevecillos que son excretados en las heces fecales. ${ }^{5}$

Es importante hacer hincapié en que la infección por E. granulosus tiene una distribución cosmopolita, y su prevalencia es muy variable dependiendo de los países e incluso dentro de cada país. Las zonas endémicas más importantes son: la cuenca mediterránea, los países de la ex-Unión Soviética, el este de África, América del Sur, zonas de China, Malasia, Sri Lanka e India. ${ }^{6}$ La información en México es limitada; se encuentran documentados en el año $1960^{7}$ cuatro casos autóctonos, dos del Distrito Federal y dos de Michoacán. En la región agropecuaria de Irapuato, Guanajuato, han sido reportados cuatro casos en 20 años, dos quistes pulmonares, un quiste hepático y un hepatobiliar, ${ }^{8}$ con un último reporte de dos casos en $2009 .{ }^{9}$

\section{CASO CLÍNICO}

Paciente femenino de 25 años de edad, originaria y residente de El Arenal, Pachuca, Hidalgo, sin antecedentes heredofamiliares de relevancia, contacto habitual con caninos, ovinos y aves silvestres, sin toxicomanías. Resección de fibroadenoma mamario izquierdo hace siete años, sin más antecedentes. Presentó cuadro de cinco meses de evolución caracterizado por dolor torácico irradiado a brazo izquierdo acompañado de tos con expectoración verdosa, síndrome febril intermitente y hemoptoico en tres ocasiones, cursando con diversos tratamientos antimicrobianos, sin mejoría.

A su ingreso, en el examen físico destacaba síndrome de condensación pulmonar con estertores crepitantes subescapulares derechos. La biometría hemática demostró leucocitos de 6,900 células $/ \mathrm{mm}^{3}$, eosinófilos de 40 células $/ \mathrm{mm}^{3}$, hemoglobina $10.7 \mathrm{~g} / \mathrm{dL}$, 
hematócrito $32.2 \%$, exámenes de función renal normal y electrocardiograma normal. Se le realizó inicialmente una radiografía de tórax que mostró una opacidad heterogénea bien delimitada en el lóbulo inferior derecho (figura 1). El estudio fue complementado con una tomografía de tórax que mostró presencia de imagen redondeada con pared engrosada y nivel hidroaéreo, con signo del «menisco» y $8.9 \mathrm{~cm}$ en su eje mayor, localizada hacia el lóbulo inferior del pulmón derecho (figuras 2 y 3 ). Ante este hallazgo radiológico, además del lugar de residencia de la paciente, se solicitaron anticuerpos para Echinococcus e inició el tratamiento empírico con albendazol y valoración por cirugía de tórax para tratamiento quirúrgico definitivo. La paciente fue abordada mediante toracotomía posterolateral con lobectomía inferior derecha, lográndose visualizar durante el procedimiento adherencias firmes de lóbulo inferior derecho a pared y diafragma, con lesión quística intraparenquimatosa (80\%). El resultado de la biopsia confirmó el diagnóstico de quiste hidatídico de $9 \times 8$ $x 6.5 \mathrm{~cm}$ (figuras 4 y 5); asimismo, fueron obtenidos anticuerpos Echinococcus IgG positivos en suero, por lo que continuó tratamiento con albendazol a dosis de $10 \mathrm{mg} / \mathrm{kg} / \mathrm{día}$. En el posoperatorio presentó quilotórax derecho, dándose manejo inicial conservador mediante nutrición parenteral y complementado mediante ligadura con clipaje del conducto torácico, ameritó estancia

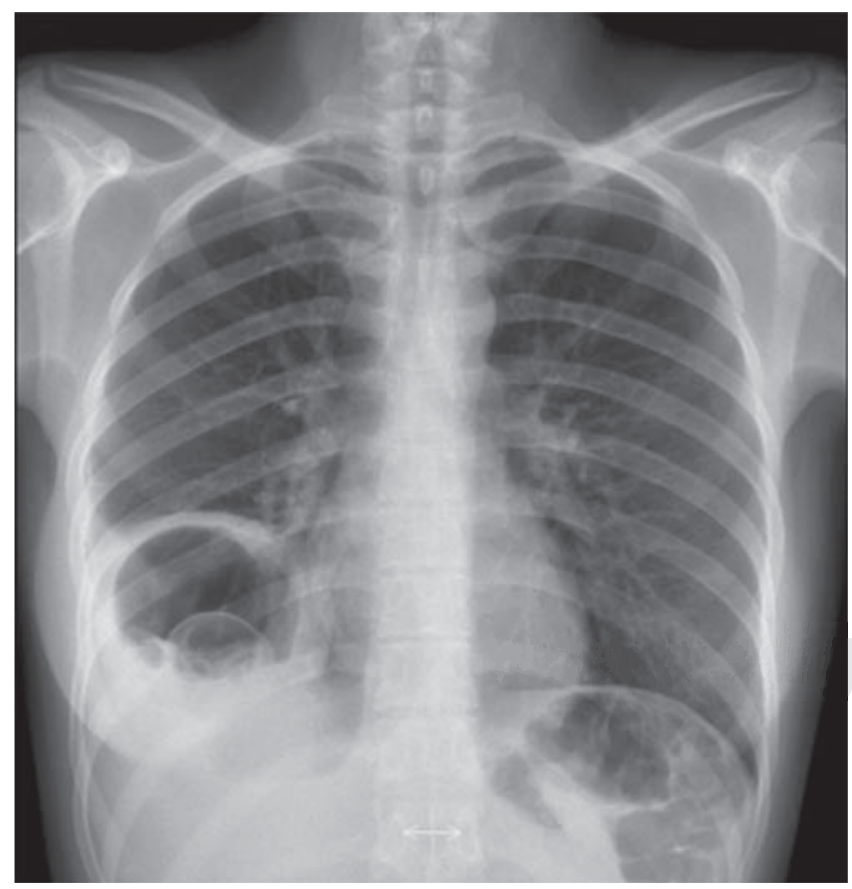

Figura 1. Radiografía de tórax con opacidad heterogénea en lóbulo inferior derecho. en la unidad de cuidados intensivos por 15 días. Se agregó coinfección por Pseudomonas aeuroginosa con sensibilidad en cultivo a tratamiento con ciprofloxacino y amikacina. Fue dada de alta en buenas condiciones.

\section{DISCUSIÓN}

El ser humano puede convertirse en un hospedador intermediario accidental al ingerir huevos de helminto;

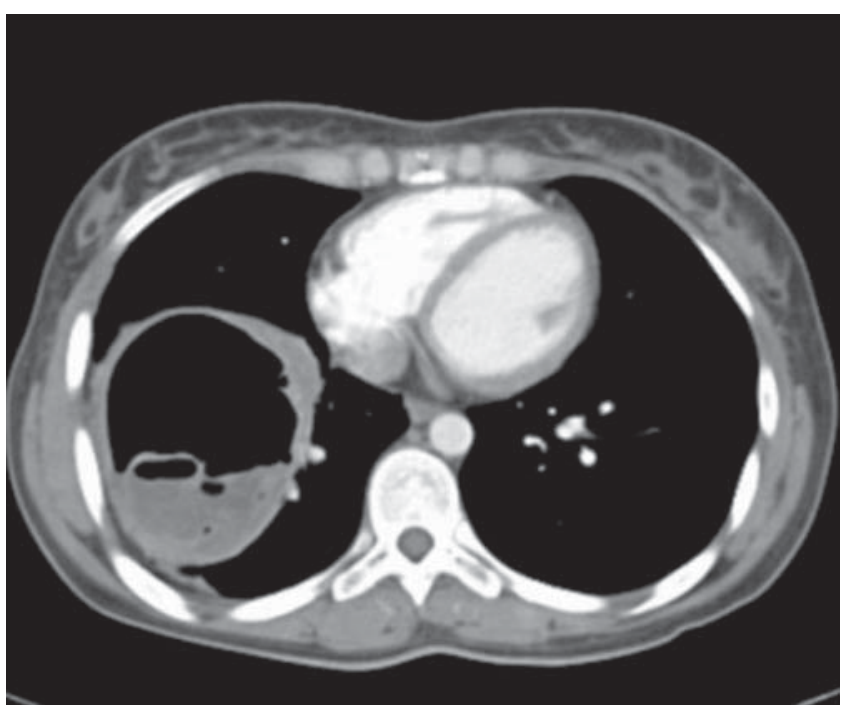

Figura 2. Tomografía axial computada de tórax con contraste. Ventana para mediastino que muestra lesión que no capta medio de contraste con signo del menisco.

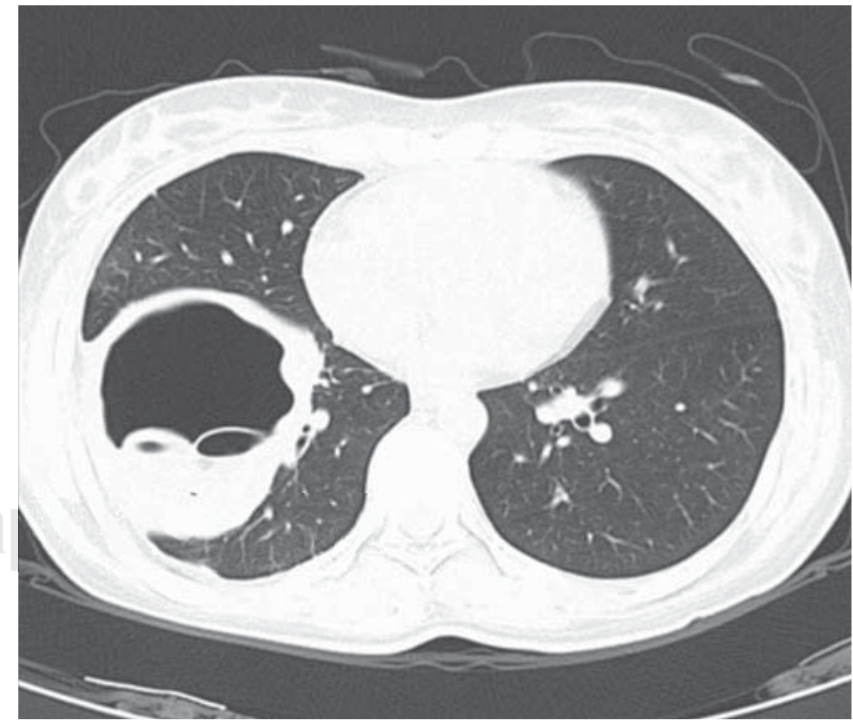

Figura 3. Tomografía axial computada de tórax. Ventana para pulmón que muestra lesión redondeada con pared engrosada y nivel hidroaéreo. 


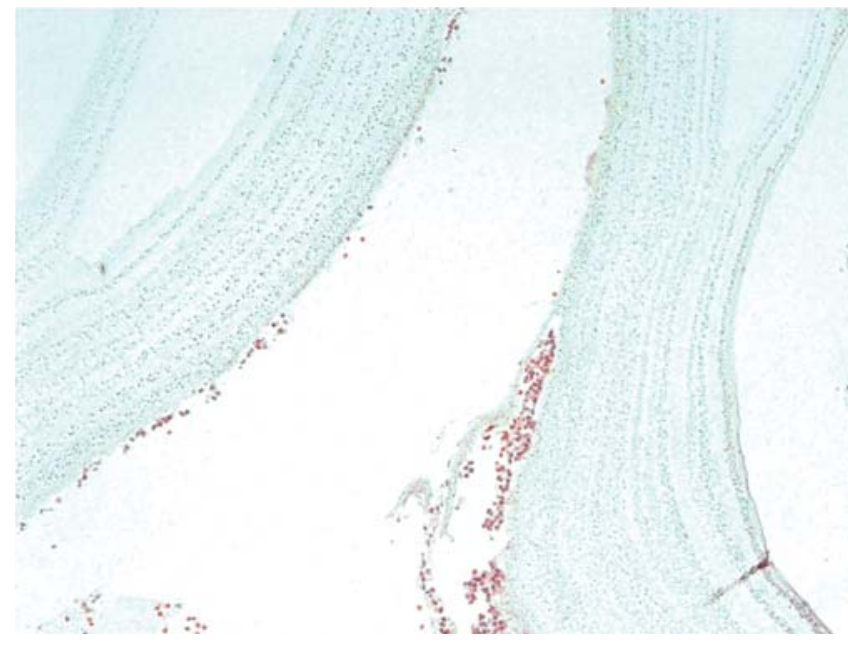

Figura 4. Detalle a bajo aumento (10x). Se observa la pared del quiste compuesta de quitina.

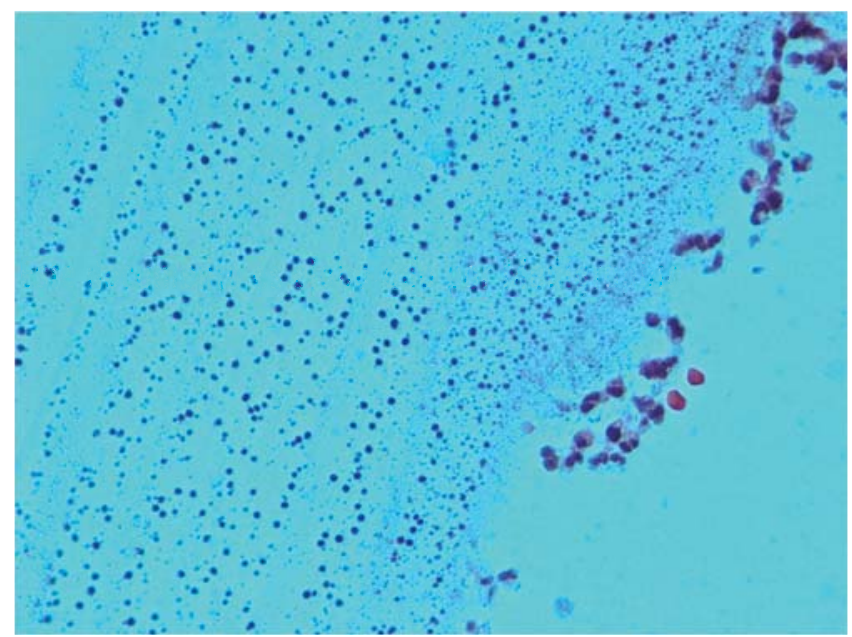

Figura 5. Detalle a alto aumento (40x). Se observa la pared del quiste de aspecto hialino.

bien directamente del hospedador definitivo o por el consumo de agua o verduras contaminadas con deposiciones de los hospedadores definitivos. Tras la ingestión, los huevos eclosionan en el intestino delgado y se liberan oncosferas que penetran en la pared intestinal. Estas oncosferas se dirigen por vía circulatoria hasta el hígado, donde se retiene la mayoría; y el resto, accede por la circulación venosa al pulmón e incluso supera el filtro pulmonar y llega a través de la circulación sistémica a otros órganos. ${ }^{6}$

El $80 \%$ de los pacientes tienen hasta un solo órgano involucrado y un quiste solitario localizado en el hígado o los pulmones. La relación hígado/pulmón puede variar de 2 a 1 hasta 7 a $1 .^{2}$ El pulmón permanece como el segundo sitio más común de involucro en adultos (después del hígado) y el sitio más común en niños. ${ }^{10} \mathrm{El}$ período de incubación entre el contagio y las manifestaciones es muy variable, desde meses hasta años. Aunque se han descrito casos en pacientes desde 1 hasta 75 años, la edad más frecuente en el área mediterránea son los 20-40 años, sin predominio en función del sexo. En general, la aparición de hidatidosis pulmonar se asocia a edades más tempranas que la forma hepática pura, situación atribuida a la mayor distensibilidad del parénquima pulmonar. Las manifestaciones clínicas dependen de la integridad o rotura del quiste, así como de las complicaciones asociadas a la rotura. Así, en la hidatidosis pulmonar no complicada lo más frecuente es la ausencia de síntomas; durante la rotura, el dato más característico es la presencia de vómica, mientras que las formas complicadas se expresan por múltiples síntomas y signos. ${ }^{6}$

Los hallazgos radiográficos y tomográficos más importantes son la presencia de lesiones quísticas que pueden ser solitarias o múltiples, unilaterales o bilaterales (20-50\%), encontrados predominantemente en los lóbulos inferiores $(60 \%)$ con un diámetro entre 1 y $20 \mathrm{~cm} .^{10}$

Los quistes no complicados pueden ser vistos como masas redondas $u$ ovaladas que tienen paredes con bordes bien definidos y refuerzan con el medio de contraste. Algunos signos clásicos como «el signo del menisco» o «signo de la media luna» se caracterizan por la presencia de aire entre el periquiste y la membrana laminada, incluso, algunos autores han llegado a considerar este fenómeno como un signo inminente de rotura. Cabe mencionar que la coexistencia de enfermedad pulmonar y hepática está presente sólo en un $6 \%$ de los casos. ${ }^{10}$ El diagnóstico es basado en la presencia de estos hallazgos clínicos, técnicas de imagen y serología (útil para confirmar el diagnóstico presuntivo, aunque algunos pacientes no demuestran una respuesta inmune detectable, con rangos de entre $50-60 \%$ para quistes pulmonares), siendo de ayuda el examen microscópico de los fluidos e histología al detectar las protoescolices. ${ }^{2}$

El tratamiento de elección, en la mayoría de las ocasiones, es quirúrgico para una resección radical del quiste. Las opciones quirúrgicas incluyen lobectomía, periquistectomía o resección en cuña del quiste, minimizando estrictamente en cada procedimiento quirúrgico el derrame del contenido del quiste a fin de evitar la diseminación intraoperatoria y recurrencia. Esto puede lograrse mediante la resección del quiste intacto o con la aspiración del líquido quístico con o sin el uso de una solución escolicida, aunado a un tratamiento preoperatorio con albendazol (10-15 mg/kg/día). Los 
agentes escolicidas más usados son formaldehído al $1 \%$ o solución salina hipertónica; aunque también pueden ser utilizados la cetrimida, polividona yodada, formalina, etanol y peróxido de hidrógeno. La terapia con albendazol se recomienda mínimo tres meses. ${ }^{4,11}$

En el caso de nuestra paciente, el diagnóstico definitivo de hidatidosis pulmonar se estableció al conjuntar la epidemiología (contacto con perros y ovinos), las manifestaciones clínicas (curso subagudo) y radiológicas (lesión única, bien delimitada, quística con signo del menisco), corroborado por histopatología y serología específica, en quien el tratamiento médico y la intervención quirúrgica se realizó de forma inmediata, con resolución de complicaciones posoperatorias. Como conclusión, podemos señalar que si bien la hidatidosis pulmonar es infrecuente en nuestro país, debe tenerse en cuenta como diagnóstico diferencial de abscesos pulmonares.

\section{REFERENCIAS}

1. Somily A, Robinson JL, Miedzinski LJ, Bhargava R, Marrie TJ. Echinococcal disease in Alberta, Canada: more than a calcified opacity. BMC Infect Dis 2005;5:34.

2. Brunetti E, Kern P, Vuitton DA; Writing Panel for the WHO-IWGE. Expert consensus for the diagnosis and treatment of cystic and alveolar echinococcosis in humans. Acta Trop 2010;114(1):1-16. doi: 10.1016/j. actatropica.2009.11.001.

3. Álvarez ZC, Riveros SP, Miranda TR, Yarur VO. Quiste hidatídico mediastínico. Reporte de un caso y revisión de la literatura. Rev Chil Infect 2007;24(2):149-152.
4. Moro P, Schantz PM. Echinococcosis: a review. Int J Infect Dis 2009;13(2):125-133. doi: 10.1016/j.ijid.2008.03.037.

5. Eroğlu A, Kürkçüoğlu C, Karaoğlanoğlu N, Tekinbaş C, Kaynar H, Onbaş O. Primary hydatid cysts of the mediastinum. Eur J Cardiothorac Surg 2002;22(4):599-601.

6. Pérez-Arellano JL, Andrade MA, López-Abán J, Carranza C, Muro A. Helmintos y aparato respiratorio. Arch Bronconeumol 2006;42(2):81-91.

7. Biagi FF, Mekbel AS. Hidatidosis humana autóctona de la República Mexicana. En: Libro homenaje al Dr. Eduardo Caballero y Caballero. México: SEP/IPN; 1960. pp. 353-356.

8. Carrada BT. Equinococosis pulmonar: investigación clínico-patológica. Med Int Mex 2004;20(1):65-71.

9. Palafox D, Palafox J, Tello B. Hidatidosis pulmonar. Reporte de dos casos clínicos. Rev Inst Nal Enf Resp Mex 2009;22(1):32-36.

10. Martínez S, Restrepo CS, Carrillo JA, et al. Thoracic manifestations of tropical parasitic infections: a pictorial review. Radiographics 2005;25(1):135-155.

11. Morar R, Feldman C. Pulmonary echinococcosis. Eur Respir J 2003;21(6):1069-1077.

\section{$\triangle$ Correspondencia:}

Dr. Alejandro Rogelio Canseco Raymundo, Instituto Nacional de Enfermedades Respiratorias Ismael Cosío Villegas, Ciudad de México.

Calzada de Tlalpan Núm. 4502, Colonia Sección

XVI. México, D.F., 14080.

Teléfono: 54-87-17-00

Correo electrónico: valentisu@hotmail.com

Los autores declaran no tener conflicto de intereses. 